\title{
Linx
}

Revue des linguistes de l'université Paris X Nanterre

$72 \mid 2015$

Former à l'écrit universitaire, un terrain pour la linguistique?

\section{Le développement des habiletés rédactionnelles à l'université : expertise discursive et acculturation aux discours universitaires}

\section{Antoine Tassin et Nathalie Spanghero-Gaillard}

\section{OpenEdition}

\section{Journals}

Édition électronique

URL : http://journals.openedition.org/linx/1648

DOI : $10.4000 /$ linx.1648

ISSN : 2118-9692

\section{Éditeur}

Presses universitaires de Paris Nanterre

Édition imprimée

Date de publication : 1 septembre 2015

Pagination : 169-188

ISSN : 0246-8743

\section{Référence électronique}

Antoine Tassin et Nathalie Spanghero-Gaillard, « Le développement des habiletés rédactionnelles à I'université : expertise discursive et acculturation aux discours universitaires », Linx [En ligne], 72 | 2015, mis en ligne le 01 mars 2016, consulté le 01 mai 2019. URL : http:// journals.openedition.org/linx/1648; DOI : 10.4000/linx.1648 


\title{
Le développement des habiletés rédactionnelles à l'université : expertise discursive et acculturation aux discours universitaires
}

\author{
Antoine TASSIN et Nathalie SPANGHERO-GAILLARD \\ Unité de recherche interdisciplinaire OCTOGONE, Université Toulouse 2, France
}

\begin{abstract}
Résumé
L'étude psycholinguistique de l'expertise rédactionnelle autour de la capacité d'adaptation d'un texte à un lectorat cible suppose que des marqueurs en soient identifiés. La formation universitaire et l'acculturation discursive qu'elle sous-tend constituent un terrain propice à l'observation du développement de cette habileté. Le cadre théorique du métadiscours, qui permet d'organiser le contenu propositionnel du discours en direction du lectorat, comprend a priori des marqueurs linguistiques de la conscience du lectorat. Les directives, comme marqueurs explicites de l'interaction avec le lectorat dans le texte, pourraient représenter une prise en charge experte du lectorat. Leur fréquence d'apparition dans des écrits scientifiques experts francophones les rend toutefois insuffisantes pour décrire l'interaction avec le lectorat. Parallèlement, les caractéristiques pragmatico-énonciatives du discours scientifique, qui correspondent aussi aux difficultés d'acculturation des étudiants, peuvent être repérées dans des procédés métadiscursifs. Ces réflexions conduisent à interroger les liens entre discours universitaires, conscience du lectorat et métadiscours.
\end{abstract}

Mots-clés

Conscience du lectorat, expertise discursive, discours universitaires, acculturation discursive, métadiscours.

\section{Introduction : tracer les contours d'une approche interdisciplinaire pour penser une formation aux écrits universitaires}

L'élaboration d'un dispositif de formation aux écrits universitaires requiert la compréhension du développement des compétences discursives nécessaires à la production de discours universitaires. Le manque de formation explicite à la rédaction universitaire oblige à reconnaitre l'existence d'un apprentissage implicite, puisque des étudiants non formés à la rédaction produisent des écrits universitaires jugés acceptables par leurs évaluateurs. Une formation explicite doit donc s'appuyer sur ce développement implicite pour le soutenir et/ou l'orienter. D'ailleurs, même sans aller jusqu'à l'enseignement spécifique de la rédaction, la prise en compte de ces éléments de réflexion paraît nécessaire à la résolution des problèmes que rencontrent les étudiants.

Comment dès lors appréhender cette évolution des habiletés rédactionnelles à l'université ? Une telle étude peut se fonder sur les démarches et apports de différentes disciplines : la psychologie cognitive, en ce qu'elle s'attache à caractériser l'expertise rédactionnelle et le développement des habiletés rédactionnelles, peut en fournir un cadre général. Puisqu'il s'agit de former à l'écriture, il apparaît pertinent d'identifier des marqueurs discursifs des habiletés cognitives décrites par la psychologie cognitive, dans une optique psycholinguistique. De tels marqueurs incarnent en effet le produit de l'expertise dans un contexte socio-discursif donné et pourraient par conséquent être particulièrement utiles dans une perspective didactique. Complémentairement, la description linguistique de discours universitaires experts, notamment sur le plan pragmatico-énonciatif, peut permettre de fixer les objectifs d'une 
formation aux discours universitaires. Enfin, cette évolution des habiletés discursives passe par une formation universitaire qui, même si elle ne s'occupe pas spécifiquement du discours, structure la pratique discursive des étudiants et autorise cette évolution. Les difficultés rencontrées par les étudiants dans l'acculturation aux pratiques discursives appropriées révèlent en même temps la progression du développement. Ce cadre demande donc à être caractérisé au niveau didactique.

Ainsi, afin de poser les jalons d'une telle approche interdisciplinaire du développement des habiletés discursives à l'université, il convient d'abord de tenter de définir l'expertise rédactionnelle et les modalités d'accès à cette expertise. Il s'agit ensuite identifier des marqueurs discursifs possibles de l'expertise dans des discours universitaires experts. En outre, une comparaison des caractéristiques pragmatico-énonciatives des discours universitaires et des difficultés des étudiants peut être effectuée. Les caractéristiques des discours universitaires regroupent, d'une part, les attentes des experts de l'université dans leur évaluation discursive des productions des étudiants (non-experts) et, d'autre part, les caractéristiques du discours scientifique (produit par ces mêmes experts). Elles peuvent donc être mises en relation avec les difficultés des étudiants à répondre à ces attentes. Cette comparaison faisant apparaître de grands axes communs, la propension des marqueurs identifiés à assurer la construction discursive de ces axes doit enfin être examinée, afin de mesurer la pertinence de ces marqueurs dans le développement des habiletés discursives.

\section{L'expertise rédactionnelle : plafonnement et pistes de dépassement}

En psychologie cognitive du langage, l'accès à l'expertise rédactionnelle a notamment été modélisé par C. Bereiter et M. Scardamalia (1987) : la pratique de l'activité rédactionnelle amène le rédacteur à passer progressivement d'une production basée sur une stratégie fonctionnelle novice (stratégie des connaissances relatées) à une production basée sur une stratégie experte (stratégie des connaissances transformées). Ce modèle ayant été validé empiriquement, le déploiement de la stratégie des connaissances transformées par le rédacteur expert est établi. Cette conception de l'expertise pose toutefois un problème certain : elle est en effet conçue comme un stade ultime de développement des habiletés rédactionnelles alors que cette stratégie serait maîtrisée dès 16-17 ans (Alamargot et Lebrave, 2010). Par conséquent, ce modèle ne permet pas de rendre compte des écarts de performance rédactionnelle entre un lycéen et un rédacteur ayant poursuivi une pratique régulière de l'activité de rédaction au-delà du lycée.

Afin de dépasser ce plafonnement de l'expertise, R.T. Kellogg (2008) propose un modèle développemental fondé sur l'évolution des stratégies fonctionnelles de production dont la maîtrise correspond à un macro-stade de développement. Ce modèle comprend une stratégie supplémentaire (stratégie des connaissances retravaillées) correspondant à un stade de développement plus expert que celui des connaissances transformées, ainsi qu'un axe supplémentaire de développement des habiletés. Il postule que l'axe de développement le plus étudié dans la littérature, l'enchaînement fonctionnel des processus cognitifs centraux impliqués dans la production écrite, permet effectivement de caractériser l'évolution des habiletés du stade des connaissances relatées à celui des connaissances transformées. Néanmoins, il ne donne que peu d'indications sur la suite du développement. Ce modèle repose ainsi sur l'hypothèse que les habiletés se développent autour de l'exploitation de trois représentations mentales tout au long de la production d'un texte :

- la représentation de l'auteur, qui contient le message que le rédacteur projette de communiquer, ses intentions communicationnelles ; 
- la représentation du texte, qui renvoie au message effectivement véhiculé par le texte en cours de production, ce que le texte signifie intrinsèquement ;

- la représentation du lectorat, qui correspond à l'anticipation par le rédacteur des réactions du lectorat visé, lors de sa lecture du texte.

Pour être exploitées, ces représentations mentales devraient être activées et maintenues en mémoire de travail de manière stable (c'est-à-dire constamment, sans fluctuation) et complète (elles doivent être suffisamment élaborées pour pouvoir orienter l'ensemble des traitements opérés par les processus centraux). D'après R.T. Kellogg, l'exploitation des représentations mentales a un coût cognitif supplémentaire sur la production : au coût déjà important des traitements opérés par les processus centraux s'ajoute celui des représentations mentales devant orienter ces traitements. Par conséquent, étant donné les capacités limitées de la mémoire de travail, plus les traitements à opérer (processus centraux et représentations mentales) seront coûteux, plus certains traitements devront être procéduralisés.

C'est notamment en ce sens que les représentations mentales ne pourraient toutes être exploitées que par l'expert avancé, car il aura, par une pratique continue de la rédaction, réalisé cette procéduralisation d'une quantité plus importante d'opérations psycholinguistiques (sur ce point, voir Alamargot, Plane, Lambert et Chesnet, 2010). Sur le plan développemental, ce modèle suggère en effet que le rédacteur novice (connaissances relatées) ne pourrait exploiter efficacement que la représentation de l'auteur : il serait essentiellement focalisé sur le message qu'il souhaite véhiculer. Le rédacteur d'expertise intermédiaire (connaissances transformées) pourrait, quant à lui, activer et comparer les représentations auteur et texte. Il parviendrait ainsi à ajuster le sens véhiculé par son texte en cours de production à ses intentions communicationnelles. Enfin, le rédacteur d'expertise avancée (connaissances retravaillées) utiliserait simultanément les trois représentations de l'auteur, du texte et du lectorat. Il serait en mesure d'activer et de maintenir ces trois représentations qui orienteraient les traitements opérés par le déploiement de l'architecture cognitive experte. Il pourrait alors, en cours de rédaction, anticiper les réactions du lectorat projeté à la lecture du texte et les prendre en charge afin d'influer sur celles-ci pour atteindre ses objectifs communicationnels. C'est donc cette capacité de prise en charge du lectorat qui caractériserait l'expertise avancée.

Cette conception de l'expertise peut s'appuyer sur différentes études ayant démontré qu'un effet significatif de l'adaptation du texte au lectorat visé ne s'observe pas chez le rédacteur avant la fin du lycée ou le début de l'université (Bracewell, Scardamalia \& Bereiter, 1978 ; Crowhurst \& Piche, 1979; Flower, 1979; Smith \& Swan, 1977). D’autres études ont également mis en évidence que des rédacteurs de première année universitaire sont encore largement focalisés sur le contenu de leur production, tandis que des rédacteurs plus avancés élaborent une représentation du lectorat qui influence fortement l'activité (Flower \& Hayes, 1980 ; Berkenkotter, 1981). Il faut cependant noter que, paradoxalement, des traces de la conscience du lectorat ont été identifiées dans des écrits de rédacteurs très jeunes, dès la fin du primaire et au début du collège (Cohen \& Riel, 1989 ; Lindgren, Leijten \& van Waes, 2011 ; Quick, 1983). Ainsi, la conscience du lectorat serait à la fois un trait caractéristique d'une expertise avancée et une capacité déjà présente chez les novices. Il apparaît de fait nécessaire d'identifier les habiletés spécifiquement expertes en matière d'adaptation du texte au lectorat.

\section{Le métadiscours : vers des marqueurs discursifs de l'adaptation experte au lectorat}

\subsection{Le métadiscours : un cadre général d'analyse linguistique du rapport au lectorat}


Dans une perspective psycholinguistique, l'identification d'habiletés expertes peut se traduire par l'identification de marqueurs de surface de cette habileté. La notion de métadiscours peut s'avérer utile à cette fin. Le métadiscours se comprend à partir de son opposition avec les contenus propositionnels. Alors que les contenus propositionnels se réfèrent au réel et véhiculent donc le contenu informatif du discours, le métadiscours se réfère au discours et relève de l'organisation des contenus propositionnels en vue de leur réception par le destinataire (Williams, 1981 ; Vande Kopple, 1985; Crismore, 1989). Ainsi défini, le métadiscours est une catégorie essentielle du discours : il participe à la réalisation de deux des trois grandes fonctions du langage (fonction textuelle et fonction interpersonnelle) identifiées par Halliday (Halliday \& Hasan, 1976; Halliday, 1985). La présence du métadiscours a d'ailleurs été observée dans de nombreux genres et pour différentes communautés : écrits d'étudiants (Hyland, 2005), articles scientifiques (Hyland, 2001 ; 2002), lettres de P.D.G. d'entreprises cotées en bourse destinées aux actionnaires (Hyland, 1998), rapports environnementaux (Skulstad, 2005), éditoriaux journalistiques (Le, 2004), descriptions de postes à pourvoir (Fu, 2012) - pour n'en citer que quelques-uns. Il excède donc largement le seul contexte universitaire et peut même, par sa fonction d'organisation du contenu propositionnel, concerner a priori tout type de discours.

Le modèle du métadiscours construit par K. Hyland (2005) constitue une classification fonctionnelle systématique des segments métadiscursifs. Il distingue le métadiscours interactif, par lequel l'auteur tient compte de l'intelligibilité de son discours par le lectorat (interaction implicite), du métadiscours interactionnel, par lequel l'auteur se situe par rapport à son propos et à son lectorat (interaction explicite). Trois pôles métadiscursifs peuvent ainsi être distingués à partir de ce modèle : l'intelligibilité du discours, le positionnement de l'auteur et l'implication du lectorat.

Le pôle intelligibilité du discours réunit cinq catégories de marqueurs :

- les marqueurs de transition établissent des relations microstructurelles entre des segments du raisonnement, notamment grâce à des connecteurs ;

- les marqueurs de cadre ordonnent (par ex. dans un premier temps), étiquettent (pour conclure) et annoncent (nous voulons montrer que) le discours en explicitant sa macrostructure ;

- les marqueurs intratextuels renvoient à des parties antérieures et postérieures du discours et guident le lectorat à travers sa macrostructure (voir infra, cf. figure 2) ;

- les marqueurs extratextuels indiquent la source d'un segment du raisonnement et ont une fonction d'étayage (Freud souligne que) ;

- enfin, les marqueurs de glose explicative développent un segment du raisonnement en le reformulant (c'est-à-dire), en l'exemplifiant (par exemple) et en l'explicitant (compris comme).

Le pôle positionnement de l'auteur, quant à lui, comprend quatre catégories :

- les marqueurs d'incertitude restreignent la portée vériconditionnelle d'un énoncé et permettent à l'auteur de s'en distancier, étendant ainsi la négociation interlocutoire de l'énoncé (il semble que) ;

- les marqueurs de certitude, renforcent le degré de conviction avec lequel une proposition est présentée par l'auteur, restreignant au contraire la négociation (clairement) ;

- les marqueurs d'opinion servent l'expression d'une position plus affective, moins épistémique de l'auteur, par le biais de commentaires plus personnels sur un énoncé (surprenant, abusivement) ;

- enfin, les mentions de soi permettent à l'auteur d'expliciter sa présence énonciative dans le discours (nous exclusif). 
Enfin, le pôle implication du lectorat rassemble cinq catégories :

- les mentions du lectorat incluent celui-ci explicitement dans le raisonnement (on, nous inclusifs) ;

- les apartés adressés au lectorat sont des méta-commentaires clairement démarqués du raisonnement en cours par lesquels l'auteur interpelle son lectorat (beaucoup d'exemples d'intersection partielle - et sinon la plupart - [...]);

- les références à des connaissances partagées invitent le lectorat à admettre une idée présentée comme communément admise (bien que l'on parle d'angioplastie d'une artère, on ne dilate bien sûr pas toute l'artère);

- les questions, réelles ou rhétoriques, attirent l'attention du lectorat sur un point donné en l'interpellant ;

- enfin, les directives enjoignent le lectorat à réaliser une action dans le but d'interpréter un segment du raisonnement (il faut souligner que).

Ainsi définis, ces trois pôles du métadiscours peuvent marquer une adaptation du texte au lectorat : au-delà de la cohérence et de la cohésion du texte, le pôle intelligibilité du discours peut indiquer la façon dont un rédacteur guide son lectorat dans sa compréhension du texte. Le pôle positionnement de l'auteur, quant à lui, peut permettre l'étude de la construction d'un ethos d'auteur, d'une légitimité d'auteur dont il faut convaincre le lectorat. Enfin, le pôle implication du lectorat peut s'analyser comme une gestion explicite du rapport à établir avec le lectorat.

Précisons qu'il ne s'agit pas de dire ici que la prise en compte du lectorat se réduit au métadiscours: les contenus propositionnels (les idées) peuvent également relever de cette conscience. Dans une argumentation, par exemple, la sélection des arguments les plus pertinents et l'anticipation et la réfutation de contre-arguments sont directement liées au lectorat cible (Coirier, Andriessen \& Chanquoy, 1999). Par extension, une faible présence du métadiscours n'implique pas nécessairement une faible conscience du lectorat. Toutefois, les contenus propositionnels susceptibles de marquer la conscience du lectorat varient d'un discours à l'autre. La contre-argumentation, comme marqueur de la conscience du lectorat, ne sera pas forcément présente et/ou pertinente dans un texte descriptif. En revanche, le métadiscours peut se retrouver d'un discours à l'autre et, même si sa distribution est variable en fonction du discours concerné, il remplira toujours cette fonction d'organisation des contenus en vue de leur réception par le destinataire. En outre, il est effectivement très présent dans les discours universitaires qui nous intéressent ici (Hyland, 2005).

Cependant, il convient de remarquer que ces procédés ne relèvent pas d'emblée d'une adaptation experte au lectorat: les connecteurs, par exemple, inclus dans les marqueurs de transition, apparaissent très tôt, dans des écrits d'élèves de primaires (Favart et Passerault, 1999). Cette observation n'exclut pas que l'utilisation de procédés, a priori plus simples, varie en fonction de l'expertise. Au demeurant, il serait pertinent d'identifier les procédés métadiscursifs à la fois fréquents dans des écrits experts et susceptibles d'être moins présents - voire absents - dans des écrits d'expertise moindre. Il apparaît ainsi raisonnable d'affirmer que le métadiscours peut constituer un terrain d'investigation pertinent de l'adaptation du texte au lectorat.

3.2. Un procédé métadiscursif expert : les directives 
La catégorie des directives peut se révéler intéressante comme marqueur d'un rapport expert au lectorat-cible. Parmi les marqueurs d'implication du lectorat, en effet, c'est elle qui a été le plus fréquemment relevée par K. Hyland (2001) dans un corpus d'articles scientifiques regroupant les sciences humaines, les sciences sociales et les sciences technologiques. Les directives sont de véritables injonctions adressées au lectorat de traiter le texte conformément à la volonté de l'auteur. Il s'agit d'énoncés directifs, réalisés par des verbes à l'impératif (considérons), des modaux d'obligation (on doit souligner) et des prédicats adjectivaux (il est nécessaire de rappeler) contrôlant un verbe d'action. Construites autour de l'injonction, de l'obligation et de la nécessité, elles sont un procédé remarquablement autoritaire - elles se combinent avec l'affirmation d'un ethos fort. Elles pourraient par conséquent marquer une prise en charge du lectorat s'apparentant à une expertise rédactionnelle avancée.

Pour exploiter les directives, il importe cependant de vérifier qu'elles constituent effectivement un procédé linguistique important dans l'interaction universitaire experte en français. En ce sens, nous avons étudié les directives sur un corpus d'articles scientifiques francophones (Tassin, 2012). L'objectif était de répliquer l'analyse automatique d'un corpus en anglais effectuée par K. Hyland (2002) sur un corpus d'articles scientifiques francophones récents, représentatif des sciences humaines et sociales. Notre corpus réunit huit disciplines (linguistique appliquée, philosophie, littérature et psychanalyse pour les sciences humaines ; marketing, sociologie, droit et économie pour les sciences sociales). Pour chaque discipline, une centaine de pages environ ont été extraites de trois revues scientifiques, pour un total de 49 articles, tous publiés entre 2001 et 2011. Les articles ont par ailleurs été sélectionnés autour de thématiques communes définies par discipline.

Les réalisations formelles possibles des directives retenues par l'étude d'origine en anglais ont été traduites extensivement : tous les équivalents français ont ainsi été conservés, à l'exception de ceux qui ne pouvaient pas intervenir dans la formation des directives telles que définies par K. Hyland. Au total, 314 formes ont été recherchées.

Sur le plan quantitatif, seules 9,1 directives par 10000 mots ont été relevées. Cette fréquence faible dans les articles scientifiques n'autorise pas à affirmer que les directives constituent un procédé auquel les experts recourent de manière privilégiée. De plus, les écarts d'une discipline à l'autre se sont avérés considérables : 18,6 au maximum pour la linguistique, contre 3,8 au minimum pour la littérature. La sociologie et la philosophie se situent autour de 12 directives par 10000 mots alors que le droit, le marketing, l'économie et la psychologie se situent autour de 6 directives par 10000 mots. Cette variabilité, associée à la faible fréquence des directives, ne permet pas de conclure à la pertinence de ce procédé comme entrée d'étude de l'interaction experte avec le lectorat en français. K. Hyland fait d'ailleurs l'hypothèse que ce procédé pourrait ne pas être sélectionné par les auteurs de par sa dimension trop autoritaire.

Toutefois, un autre résultat qui retient l'attention réside en d'autres énoncés pertinents qui ont été observés en marge de l'analyse automatique. La détermination a priori des réalisations formelles possibles d'un procédé, nécessaire à un traitement automatique du corpus, a en effet eu pour résultat d'écarter des énoncés correspondant pourtant à la définition du procédé. Par exemple, si cette méthode a conduit à l'extraction des directives réalisées par le verbe impersonnel falloir associé à une action (il faut noter), les énoncés construits par l'utilisation impersonnel du verbe convenir (il convient de noter), pourtant très proches à tous points de vue, n'ont pas été relevés. Cette première limite, inhérente au traitement automatique de corpus, en a fait apparaître une deuxième, relative cette fois à la définition fonctionnelle et 
formelle du procédé. Pour illustrer cette deuxième limite, analysons les trois énoncés suivants :

(1) on doit noter que ...

(2) on peut noter que ...

(3) à noter que ...

A partir de la définition des directives de K. Hyland, seul l'énoncé (1) sera retenu. Les énoncés (2) et (3), parce qu'ils ne s'interprètent pas comme relevant de l'injonction, de l'obligation ou de la nécessité, ne correspondent pas à la définition d'une directive. Pourtant, ces trois énoncés sont proches sur le plan pragmatico-énonciatif : ils invitent le lectorat, inclus par le pronom indéfini, à réaliser l'action cognitive de noter le contenu propositionnel décrit par la complétive. Autrement dit, par ces énoncés métadiscursifs, l'auteur exhorte le lectorat à focaliser son attention sur l'élément visé du raisonnement.

Il apparait par conséquent que ce procédé doit être redéfini autour de la notion d'exhortation à réaliser une action adressée au lectorat, l'objectif étant pour l'auteur que son lectorat interprète un segment de la réflexion conformément à ses intentions. Cette redéfinition ne peut passer que par une analyse manuelle de corpus afin d'en étudier toutes les réalisations possibles. Ce n'est qu'à ces conditions que le procédé pourra être examiné en vue de déterminer s'il peut constituer un marqueur pertinent d'une adaptation experte au lectorat dans les discours universitaires. Néanmoins et dans une perspective plus générale, le métadiscours dans son ensemble, c'est-à-dire avec toutes ses catégories fonctionnelles, peut permettre de caractériser l'adaptation au lectorat à différents niveaux.

\section{Discours universitaires et difficultés des étudiants : acculturation et métadiscours}

L'accès à l'expertise rédactionnelle avancée, telle que définie supra, passe par une pratique continue de l'activité rédactionnelle : la formation universitaire requiert une telle pratique et constitue sans doute l'ancrage praxéologique le plus évident du développement des habiletés rédactionnelles. Puisqu'à la fin du lycée, le rédacteur est supposé avoir atteint un certain niveau d'expertise, l'université peut être un terrain d'investigation privilégié du développement rédactionnel continu, de l'expertise intermédiaire vers l'expertise avancée.

Le développement des habiletés discursives à l'université passe par une acculturation aux pratiques discursives universitaires (Pollet et Boch, 2002). Ces pratiques sont définies par (et par rapport à) une communauté discursive disciplinaire, composée essentiellement par les chercheurs qui contribuent à la construire (Swales, 1990). L'étiquette des discours universitaires englobe un ensemble hétérogène de pratiques (des écrits attendus des étudiants aux différents écrits des chercheurs). Il faut toutefois souligner que les écrits universitaires possèdent des caractéristiques générales communes, que F. Rinck (2011) met en lien avec les difficultés rencontrées par les étudiants dans ce processus d'acculturation. A partir de trois études portant sur les caractéristiques des discours universitaires et/ou sur les difficultés des étudiants (Deschepper et Thyrion, 2008 ; Rinck, 2011 ; Rinck et Sitri, 2012), il est possible d'en identifier, schématiquement, trois grands axes: la posture de l'auteur, la conduite textuelle et l'adaptation à une communauté discursive dans laquelle le discours doit s'intégrer. Ici, il importe de voir que ces axes peuvent être réalisés, au moins partiellement, par des catégories de métadiscours.

Ainsi, la posture de l'auteur attendue dans les discours universitaires passe par un effacement énonciatif de ce dernier aux fins d'objectivation de la réflexion. Le sujet personnel doit faire 
place au sujet épistémique, faisant primer l'objet qu'il traite. Toutefois, cet effacement est à relativiser par la nécessaire prise en charge de son discours par l'auteur, qui doit développer une voix propre et un positionnement critique. Cette posture d'auteur peut être construite via le métadiscours : une gestion adaptée des mentions de soi permet à l'auteur de s'effacer et de se manifester avec pertinence. Les marqueurs de certitude et d'incertitude permettent en outre de nuancer la distance prise avec les énoncés. Les marqueurs d'opinion sont, quant à eux, à utiliser avec parcimonie.

La conduite textuelle consiste en l'élaboration d'un texte explicatif et argumentatif cohérent, c'est-à-dire un texte compréhensible et convaincant, avec des intentions claires - la cohérence et l'argumentation posant notamment problème aux étudiants. Un tel texte peut s'appuyer sur des marqueurs de transition spécifiant les relations entre les arguments et sur des marqueurs de glose explicative pour assurer une lisibilité optimale des idées développées. De plus, des marqueurs de cadre et des marqueurs intratextuels peuvent permettre l'élaboration de la cohérence globale du texte (en détaillant sa macrostructure) et l'explicitation des intentions de l'auteur (par ex. Il s'agira d'abord de montrer que).

L'adaptation à une communauté discursive coïncide en fait avec l'adaptation au lectorat du discours : en effet, un écrit d'étudiant est évalué par un enseignant-chercheur et un écrit de chercheur a vocation, au moins en partie, à être lu par d'autres chercheurs. C'est en ce sens que J.E. Porter (1992) définit le lectorat comme une abstraction, construite par le rédacteur au fur et à mesure de son acculturation à une communauté discursive donnée. S'adresser à un tel lectorat, multiple et critique, suppose de se situer par rapport à des recherches antérieures, ce qui implique un ancrage épistémique du discours. Il s'agit donc de se positionner par rapport à des idées et par rapport aux acteurs de la discipline qui co-construisent ces idées. L'adoption de cette démarche peut s'appuyer sur des marqueurs extratextuels afin de reconstituer le réseau conceptuel sur lequel la réflexion se fonde. L'utilisation des marqueurs d'implication du lectorat peut permettre à l'auteur de s'adresser à la communauté discursive afin de solliciter sa coopération dialogique et de reconnaître son rôle de critique potentiel (Hyland, 2001).

La séparation entre ces trois axes de caractéristiques des discours universitaires et de difficultés des étudiants est bien sûr artificielle : ces axes se recoupent (le positionnement de l'auteur et l'argumentation ne pouvant, par exemple, être séparés de l'adaptation à une communauté discursive). De même, les catégories de métadiscours pouvant contribuer à l'élaboration discursive de ces axes n'ont pas nécessairement une fonction unique. Par exemple, un connecteur concessif (même si) peut renforcer l'intelligibilité d'une relation entre deux segments du raisonnement, tout en assurant la gestion de la contre-argumentation et l'ancrage épistémique du discours par rapport à des auteurs dont les conclusions divergeraient. Toutefois, une attention particulière doit être portée à la dimension fonctionnelle du métadiscours en ce qu'elle met en lumière la façon dont ces différents aspects des discours universitaires sont effectivement réalisés dans les textes.

D'ailleurs, K. Hyland (2005) a pu montrer l'importance quantitative du métadiscours dans l'acculturation aux discours universitaires. Il a en effet constitué un corpus composé de 240 mémoires et thèses écrites en anglais langue étrangère par des étudiants en Master et Doctorat dans six disciplines (linguistique appliquée, administration publique, études de commerce, informatique, ingénierie électronique et biologie). Quoiqu'il s'agisse d'écrits différents, ces deux genres appartiennent aux écrits de recherche en formation (Reuter, 2004) et sont, à ce titre, comparables. K. Hyland a observé que les écrits des étudiants en Master comportaient en moyenne 378,3 segments métadiscursifs par 10000 mots et que ce chiffre passait à 508,2 
pour les étudiants en doctorat. Cette évolution considérable suggère que l'acculturation aux discours universitaires peut être analysée autour du métadiscours.

Dans une perspective différente, quoique complémentaire, les liens entre le métadiscours et l'évaluation universitaire d'écrits étudiants ont été examinés. Une première étude (Intaraprawat \& Steffensen, 1995), portant sur des textes argumentatifs produits par des étudiants en anglais langue étrangère, a montré que les productions les mieux notées comportaient davantage de métadiscours, utilisé de manière plus variée. Une deuxième étude (Cheng et Steffensen, 1996) sur une intervention didactique durant seize semaines a été réalisée. Elle opposait un groupe formé spécifiquement à l'utilisation du métadiscours et un groupe recevant un cours de rédaction traditionnel : il est apparu que le groupe métadiscours obtenait de meilleures notes et produisait davantage de métadiscours que le groupe contrôle. Ces deux études concernaient certes l'anglais langue étrangère. Toutefois, cette corrélation systématique entre une meilleure évaluation universitaire et une utilisation plus importante et plus efficace du métadiscours renforce l'idée que l'acculturation aux discours universitaires peut se construire autour du métadiscours. Elle invite en outre à considérer le lien consubstantiel que décrit M. Carter (2007) entre l'acquisition des connaissances, des démarches intellectuelles et des compétences discursives. Le métadiscours, qui relève à la fois de l'acculturation aux pratiques discursives universitaires et d'une adaptation au lectorat, composé ici par la communauté discursive, constitue ainsi une base de réflexion pour l'élaboration pluridisciplinaire d'un dispositif de formation aux écrits universitaires.

\section{Conclusion : former à l'expertise discursive pour favoriser l'acculturation aux discours universitaires}

La capacité du rédacteur à adapter son texte à son lectorat-cible pourrait constituer une voie d'accès à l'expertise rédactionnelle avancée à l'université. Une approche psycholinguistique du développement des habiletés rédactionnelles pourrait permettre de caractériser plus finement cette habileté et ses différentes étapes de développement et, par là-même, d'observer la façon dont cette compétence s'exerce dans la matérialité du discours. Une telle approche ne semble cependant pas pouvoir s'affranchir de la description didactique des différents aspects et étapes de la formation universitaire. Seule cette description pourra permettre l'étude du parcours qui permet le développement des compétences discursives d'une part et l'élaboration d'un dispositif de soutien d'autre part. De même, l'acculturation aux discours universitaires ne semble pas pouvoir être séparée du développement de la capacité à s'adapter au lectorat universitaire, car il renvoie en fait à la communauté discursive ; or c'est nécessairement par rapport à cette communauté que sont définies les pratiques discursives de l'université, pratiques auxquelles l'étudiant doit s'acculturer.

Une entrée pertinente pour relier la perspective hors contexte de l'expertise rédactionnelle et la perspective en contexte de l'acculturation aux pratiques discursives universitaires peut consister en une réflexion autour du métadiscours et de ses fonctions. L'expertise rédactionnelle devient nécessairement une expertise discursive dès lors qu'est considérée la dimension implicite de l'apprentissage de la rédaction à l'université. Expliciter l'implicite pourrait s'avérer utile en vue de favoriser cet apprentissage.

Toutefois, cette réflexion autour du métadiscours doit pouvoir s'appuyer sur des recherches plus étendues. Ainsi, l'analyse de l'ensemble du métadiscours dans des écrits universitaires en français langue maternelle (des productions des étudiants aux articles scientifiques, écrits experts de l'université) renforcerait sans doute la description linguistique et didactique de ces 
écrits. L'étude expérimentale de l'évolution de la capacité d'adaptation au lectorat sur un continuum universitaire autoriserait certainement une meilleure compréhension de l'accès à l'expertise discursive. Une telle étude expérimentale devrait tenir compte des compétences sanctionnées par les diplômes et des exercices rédactionnels par lesquels les étudiants se forment à l'écrit. L'approche décrite ici, d'emblée interdisciplinaire, paraît de nature à contribuer à une formation aux écrits universitaires.

\section{Bibliographie}

ALAMARGOT, D., LEBRAVE J.-L., 2010, "The study of professional writing: a joint contribution from cognitive psychology and genetic criticism”, European Psychologist, 15-1, p. 12-22.

ALAMARGOT D., PlANE, S., LAMBERT, E., CHESNET, D., 2010, "Using eye and pen movements to trace the development of writing expertise : case studies of a 7th, 9th and 12th grader, graduate student, and professional writer", Reading and Writing, 23-7, p. 853-888.

BEREITER, C., SCARDAMALIA, M. 1987, The psychology of written composition, Hillsdale, NJ, Lawrence Erlbaum Associates.

BERKENKOTTER, C., 1981, "Understanding a writer's awareness of audience”, College Composition and Communication, 32, p. 388-399.

BRACEWELL, R., SCARDAMALIA, M., BEREITER, C., 1978, The development of audience awareness in writing, Toronto, Canada.

CARTER, M., 2007, "Ways of knowing, doing and writing in the disciplines", College composition and communication, 58-3, p. 385-418.

CHENG, X., STEFFENSEN, M.S., 1996, "Metadiscourse : A Technique for Improving Student Writing", Research in the Teaching of English, 30-2, p. 149-181.

Cohen M., Riel, M., 1989, “The Effect of Distant Audiences on Students' Writing”, American Educational Research Journal, 26-2, p. 143-159.

COIRIER, P., CHANQUOY, L., ANDRIESSEN, J., 1999., "From Planning to Translating : The Specificity of Argumentative Writing", dans P. Coirier, J. Andriessen (dir.) Foundations of argumentative text processing, Amsterdam, University of Amsterdam, p. 1-28.

CRISMORE, A., 1989, Talking with Readers: Metadiscourse as Rhetorical Act, New York, Peter Lang Publishers.

CROWHURST, M., PICHE, G. L., 1979, "Audience and mode of discourse effects on syntactic complexity in writing at two grade levels", Research in the Teaching of English, 13, p. 101-109.

DESCHEPPER, C., THYRION, F., 2008, «L'entrée dans le supérieur et l'accès aux discours universitaires: opérationnaliser la notion de rapport à l'écrit dans un projet de formation », dans S. Chartrand, C. Blaser (dir.) Le rapport à l'écrit: un outil pour enseigner de l'école à l'université, Dyptique, 12, Namur, Presses universitaires de Namur, p. 61-86.

FAVART, M., PASSERAULT, J.M., 1999, «Aspects textuels du fonctionnement et du développement des connecteurs : approche en production », L'Année Psychologique, 99, p. 149-173.

FLOWER, L., 1979, "Writer-based prose : A cognitive basis for problems in writing", College English, 41-1, p. 19-37.

FLOWER, L., HAYES, J. R., 1980, “The cognition of discovery : Defining a rhetorical problem", College composition and communication, 31-1, p. 21-32.

FU, X., 2012, "The use of interactional metadiscourse in job postings", Discourse Studies, 144, p. 399-417.

HALLIDAY, M.A.K., 1985, Introduction to Functional Grammar, London, Edward Arnold. 
HALLIDAY, M.A.K, HASAN, R., 1976, Cohesion in English, London, Longman.

HYLAND, K., 1998, "Exploring corporate rhetoric: metadiscourse in the CEO's letter", Journal of Business Communication, 35-2, p. 224-244.

HYLAND, K., 2001, "Bringing in the reader : Addressee features in academic articles", Written communication, 18, p. 549-574.

HYLAND, K., 2002, "Directives : Argument and Engagement in Academic Writing”, Applied Linguistics, 23-2, p. 215-239.

HYLAND, K., 2005, Metadiscourse : Exploring Interaction in Writing, London, Continuum. Intaraprawat, P., Steffensen, M. S., 1995, "The use of metadiscourse in good and poor ESL essays", Journal of Second Language Writing, 4-3, p. 253-272.

KELLOGG, R.T., 2008, "Training writing skills : A cognitive developmental perspective", Journal of writing research, 1-1, p. 1-26.

LE, E., 2004, "Active participation within written argumentation: Metadiscourse and editorialist's authority", J ournal of Pragmatics, 36, p. 687-714.

LINDGREN, E., LEIJTEN, M., VAN WAES, L., 2011, "Adapting to the reader during writing", Written Language \& Literacy, 14-2, p. 188-223.

POLLET, M.C., BOCH, F., (dir.), 2002, L'écrit dans l'enseignement supérieur, Enjeux, 5354, Namur, Cedocef.

PORTER, J.E., 1992, Audience and rhetoric, Englewood Cliffs, NJ, Prentice-Hall.

QUICK, D., 1983, "Audience awareness and adoption skill of writers at four different grade levels", Dissertation Abstracts International, 44 07A (University Microfilms No. DA83225612)

REUTER, Y., 2004, «Analyser les problèmes de l'écriture de recherche en formation », Pratiques, 121-122, p. 9-27.

RINCK, F., 2011, «Former à (et par) l'écrit de recherche. Quels enjeux, quelles exigences ? », Le français aujourd'hui, 174-3, p. 79-89.

RINCK, F., SITRI, F., 2012, «Pour une formation linguistique aux écrits professionnels », Pratiques, 152-153, p. 71-84.

SKULSTAD, A.S., 2005, "The use of metadiscourse in introductory sections of a new genre", International Journal of Applied Linguistics, 15-1, p. 71-86.

SMITH, W., SWAN, M., 1977, "Adjusting syntactic structures to varied levels of audience", Journal of Experimental Education, 46, p. 29-34.

SWALES, J. M., 1990, Genre analysis: English in academic and research settings, Cambridge, Cambridge university press.

TASSIN A., 2012, De l'expertise à la super expertise rédactionnelle : étude des marques linguistiques de la prise en charge du lecteur dans l'argumentation universitaire, Mémoire de Master 2, Université de Toulouse 2-Le Mirail.

VANDE KOPPLE, W.J., 1985, "Some explanatory discourse on metadiscourse", College Composition and Communication, 36, p. 82-93.

WILLIAMS, J.M., 1981, Style: Ten Lessons in Clarity and Grace, Glenview, Scott, Foresman. 